\title{
Association of perioperative allogeneic blood transfusion with postoperative survival of patients with gastric cancer after curative gastrectomy
}

\author{
Suliang $\mathrm{Li}^{1}$, Yang $\mathrm{Ji}^{2}$, Yun $\mathrm{Ye}^{1 *}$ \\ ${ }^{1}$ Department of Blood Transfusion, the First Affiliated Hospital of Xi'an Medical University, Xi'an, Shaanxi 710077, China; \\ ${ }^{2}$ Department of Radiology, the First Affiliated Hospital of Xi'an Medical University, Xi'an, Shaanxi 710077, China
}

\begin{abstract}
To explore the association of perioperative allogeneic blood transfusion (PABT) and postoperative survival rates in patients with gastric cancer (GC). In total, 186 patients with gastric cancer accepted curative gastrectomy were divided into three groups, the PABT $<2 \mathrm{U}$ group, the PABT $>2 \mathrm{U}$ group, and the no blood transfusion group. The relationships between PABT and clinical pathological parameters were analyzed and their effects on the postoperative survival of patients with GC were studied. Multivariable COX regression results showed that PABT and tumor size are the important factors influencing the postoperative survival of patients with GC $(P<0.05)$. When comparing with the no blood transfusion group and the PABT $<2 \mathrm{U}$ group, the patient's risk of death in the PABT $>2 \mathrm{U}$ group rises to 3.282 and 2.130 times (95\%CI: $1.731-4.886$ and $1.194-3.797)(P<0.05)$, respectively. This study suggests that PABT is significantly associated with postoperative survival after curative gastrectomy in patients with GC.
\end{abstract}

Keywords: gastric cancer, perioperative allogeneic blood transfusion, survival rates

\section{INTRODUCTION}

Gastric cancer (GC) is one of the most common malignant tumors, it has high morbidity and mortality and can seriously affect the patient's health ${ }^{[1,2]}$. Surgery is critical for GC treatment, and this often induces a significant amount of intra-operative blood loss and a related need for perioperative allogeneic blood transfusion $(\mathrm{PABT})^{[3]}$. However, blood transfusion is a risk, especially for tumor patients because it can lead to immunosuppression which affects the prognosis of tumor patients ${ }^{[4]}$. In recent years, some researchers found that PABT had certain relevance to the adverse

*Correspondence to: Yun Ye, Department of Blood Transfusion, The First Affiliated Hospital of Xi'an Medical University, 48 Fenggao West Road, Xi'an, Shaanxi 710077, China. Tel:0086-29-84277552; Fax:0086-29-84277393; E-mail: yeyun236@163.com. postoperative prognosis of those with diseases such as lung cancer, liver cancer, and breast cancer ${ }^{[5,6]}$. However, controversies on the relationship between blood transfusion and poor prognosis are still far from being resolved $^{[7-9]}$. Here we adopted the method of survival analysis to explore the effect of perioperative blood transfusions on survival rates of postoperative GC.

\section{MATERIALS AND METHODS}

\section{Patients}

There were 217 patients who underwent curative resection and histologically confirmed gastric carcinoma from June 2006 to December 2012 at the Department of Oncology Surgery, the First Affiliated Hospital of Xi'an Medical University, all the patients with pathological findings of serosal involvement or nodal metastases were potentially eligible for adjuvant chemotherapy. The patients whose data 
were incomplete or displayed preoperative infection were excluded. Finally, 186 cases were included in the study, 122 cases of men and 64 cases of women, aged 25-81 years, all diagnosed with primary gastric cancer by pathology postoperatively. Collected data included patients'age, gender, BMI, preoperative anemia, tumor size, tumor site, histological grading, ascular/lymphatic neural invasion, type of gastrectomy, TNM staging, resected lymph node, presence of blood transfusion and transfusion volume. PABT was defined as either whole blood or packed red blood cells administered between 7 days before surgery and 7 days after surgery. Usually, a hemoglobin concentration of less than $100 \mathrm{~g} / \mathrm{L}$ ( $<60 \mathrm{~g} / \mathrm{L}$, transfusions will be done; 60-100 g/L, transfusions will be done depending on the discretion of the anesthetist and the surgical team responsible for the care of the patient) or significant abnormal hemodynamics intraoperatively or postoperative blood loss was used as a threshold for transfusion. TNM staging refers to the 7th of American Joint Committee on Cancer (AJCC) ${ }^{[10]}$. The study protocol was approved by the ethics committee of our institution, and all patients provided written informed consent.

\section{Case groups}

According to whether the patients accepted transfusion and the volume of blood transfused perioperatively, the patients were assigned to the PABT $<2 \mathrm{U}$ group (group A, 65 cases), the PABT $>2 \mathrm{U}$ group (group $\mathrm{B}, 43$ cases), the no blood transfusion group (group $\mathrm{C}$, 78 cases). All patients were followed up via telephone, letter or a follow-up visit. Follow-up results were expressed as survival months from the operation to death or living for the expiration of 60 months (Fig.1).

\section{Statistical analysis}

Statistical analyses were performed with SPSS

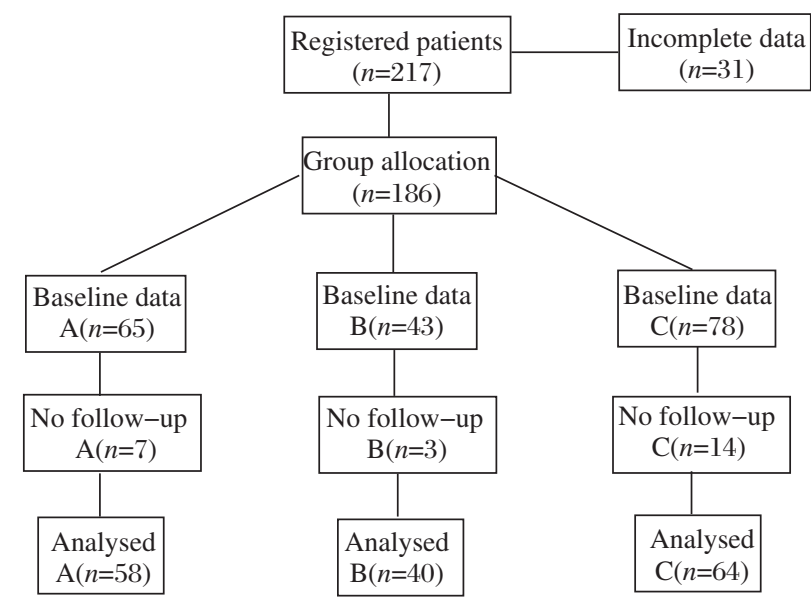

Fig.1. Trial profile. No follow-up relates to those patients where there was no information on the primary end point.
18.0 software. Descriptive results of continuous variables followed for normal distribution were presented as Mean \pm SD. A comparison among the three groups was made using the one-way ANOVA test. Categorical variables were presented as $n(\%)$ and assessed by $\chi^{2}$ test or Fisher's exact test. Kaplan-Meier was adopted for survival analysis and the survival curve was drawn. Log-rank was used to test the significance of differences among the three groups. COX regression was used to analyze the influence factors of survival of postoperative gastric cancer patients. All $P$-values are two-sided and values lower than 0.05 were considered as statistically significant.

\section{RESULTS}

\section{Incidence of perioperative blood transfusion}

There were 108 patients with GC (out of 186 cases) who underwent PABT, the incidence was $58.06 \%$, and the incidence of blood transfusion $>2 \mathrm{U}$ was $23.12 \%$.

\section{The relationships of perioperative blood transfusion and clinical pathologic features}

The differences of tumor size, ascular/lymphatic neural invasion, preoperative anemia, TNM staging, resected lymph node were found statistically significant among three groups $(P<0.05)$. On other clinical characteristics such as gender, age, BMI, tumor site, histological grading, type of gastrectomy, the differences among three groups were not statistically significant $(P>0.05)$ (Table 1).

\section{Survival time of each group and pairwise comparisons}

We successfully followed up 162 patients, including group A, 58 cases; group B, 40 cases; group C, 64 cases, the follow-up rate was $87.1 \%$. Among them, 98 cases accepted PABT(Fig.1). There were 113 of 162 patients died during follow-up: group A, 44 cases; group B, 32 cases; group $\mathrm{C}, 37$ cases. The median of survival time in the three groups were 20, 12, 27 months (Fig.2) and the $95 \%$ confidence interval $(95 \% \mathrm{CI})$ was $(16,24)$, $(10,14),(26,28)$, respectively. Log rank test was used to analyze the difference of survival time in the three groups $\left(\chi^{2}=12.797, P=0.002\right)$. The results of pairwise comparison showed that the differences between groups were all statistically significant (Table 2).

\section{The relationship between perioperative blood transfusion and postoperative survival}

Preoperative anemia, tumor size, ascular/lymphatic neural invasion, TNM staging, resected lymph node 
Table 1 Baseline data

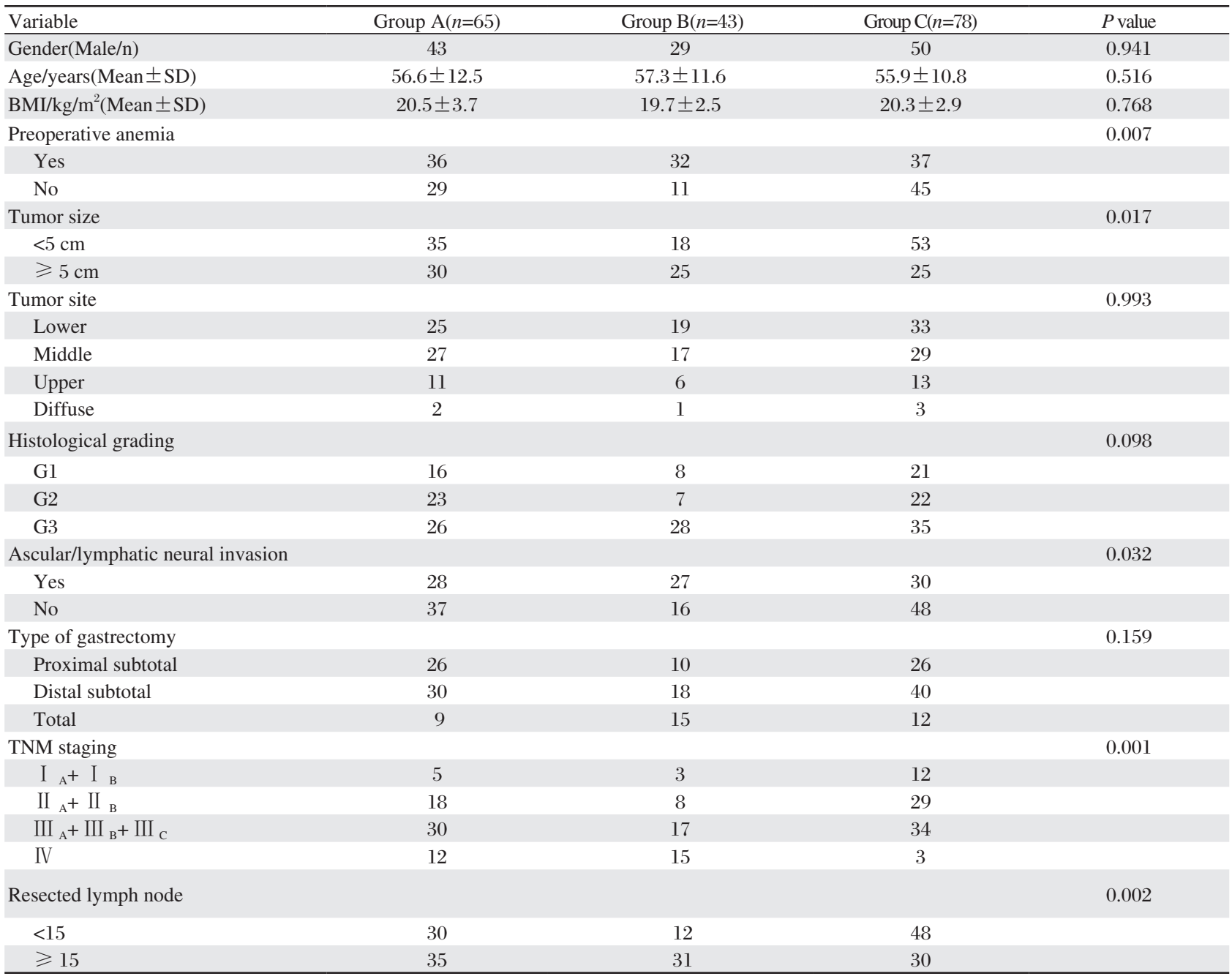

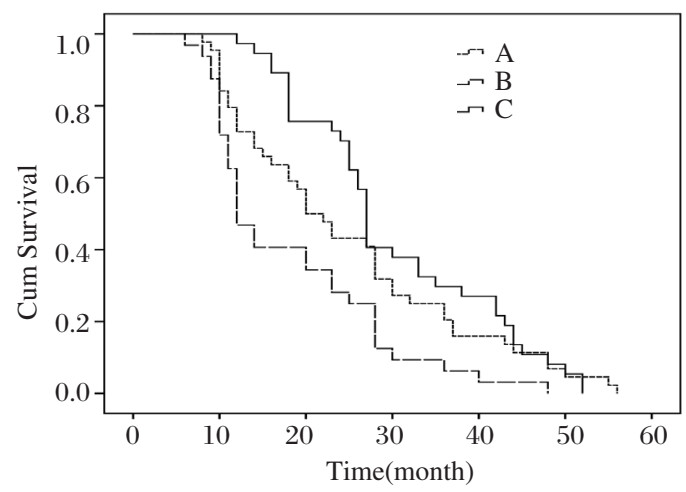

Fig.2. Cumulative survival curves of 162 patients with gastric cancer who underwent curative lgastrectomy were included in a multiariable COX regression model. The results showed that tumor size and PABT are two important factors influencing the survival of postoperative gastric cancer patients, their effects on postoperative survival rate of GC patients being statistical significant $(P<0.05)$. The patient's risk of death whose tumor size $>5 \mathrm{~cm}$ is 2.427 times of those with tumor size $<5 \mathrm{~cm}(95 \% \mathrm{CI}: 1.083-5.989)$; and the patient's risk of death whose PABT $>2 \mathrm{U}$ is 3.282 times of those with no blood transfusion (95\%CI: 1.731-4.886) and is 2.130 times of those with PABT $<2 \mathrm{U}(95 \% \mathrm{CI}$ : 1.194-3.797)(Table 3).

Table 2 Medians for survival time and comparisons between groups

\begin{tabular}{|c|c|c|c|c|c|c|}
\hline \multirow{2}{*}{ Groups } & \multicolumn{3}{|c|}{ Median } & \multirow{2}{*}{ Groups } & \multicolumn{2}{|c|}{ Log-rank } \\
\hline & Estimate & Std. Error & $95 \% \mathrm{CI}$ & & $\chi^{2}$ & $P$ value \\
\hline A & 20 & 2.211 & $15.666-24.334$ & A vs. C & 4.987 & 0.026 \\
\hline B & 12 & 1.210 & $9.629-14.371$ & $\mathrm{~B} v s . \mathrm{A}$ & 5.217 & 0.022 \\
\hline C & 27 & 0.498 & $26.024-27.976$ & Cvs. B & 4.044 & 0.044 \\
\hline Overall & 23 & 1.992 & $19.095-26.905$ & Overall & 12.797 & 0.002 \\
\hline
\end{tabular}

95\% CI, 95\% confidence interval. 
with gastric cancer after curative gastrectomy, 2017, 1(2)

Table 3 The results of multivariable COX regression analysis

\begin{tabular}{lcccccc}
\hline Variable & B & SE & Wald & Exp(B) & $95 \%$ CI & $P$ value \\
\hline PA(Yes/No) & -0.336 & 0.397 & 0.715 & 0.715 & $0.328-1.557$ & 0.398 \\
$\mathrm{TS}(\geqslant 5 \mathrm{~cm} /<5 \mathrm{~cm})$ & 0.887 & 0.461 & 3.700 & 2.427 & $1.083-5.989$ & 0.043 \\
$\mathrm{~A} / \mathrm{LNI}($ Yes/No) & 0.335 & 0.367 & 0.833 & 1.398 & $0.681-2.871$ & 0.361 \\
$\mathrm{TNM}(\mathrm{III}+\mathrm{IV} / \mathrm{I}+\mathrm{II})$ & 0.186 & 0.431 & 0.186 & 1.204 & $0.517-2.805$ & 0.666 \\
$\mathrm{RLN}(\geqslant 15 /<15)$ & 0.032 & 0.609 & 0.003 & 1.033 & $0.313-3.405$ & 0.757 \\
Transfusion & & & & & & \\
$\quad$ Group B /Group C & 1.085 & 0.427 & 8.027 & 3.282 & $1.731-4.886$ & 0.002 \\
$\quad$ Group B/Group A & 0.756 & 0.295 & 6.563 & 2.130 & $1.194-3.797$ & 0.010 \\
\hline
\end{tabular}

PA, Preoperative anemia; TS, Tumor size; A/LNI, Ascular/lymphatic neural invasion; TNM, TNM staging; RLN, Resected lymph node; RR, relative risk; $95 \% \mathrm{CI}, 95 \%$ confidence interval.

\section{DISCUSSION}

Gastric cancer is the most common gastrointestinal tumor, with gastric resection the preferred treatment ${ }^{[11,12]}$. This often brings about a significant amount of intra-operative blood loss and a consequent need for perioperative blood transfusion ${ }^{[13]}$. However, PABT may cause blood-borne diseases, blood transfusion reactions and complications such as hemolytic reaction, and therefore influences the postoperative survival rate in patients with gastric cancer ${ }^{[14,15]}$.

Our results show that the incidence of PABT in the patients with GC was $58.06 \%(108 / 186)$, and the incidence of blood transfusion amount $>2 \mathrm{U}$ was $23.12 \%(43 / 186)$. The incidence of perioperative blood transfusion and transfusion volume increased significantly with the increasing of tumor size, histological grading, ascular/lymphatic neural invasion, TNM staging and resected lymph node $(P<0.05)$. This may be caused by the wider range of operation, longer operating time and increased intraoperative blood loss in patients with middle and advanced stage cancer. In addition, the other underlying reasons of blood transfusion are the relatively late confirmed diagnosis time and a certain extent of anemia exist before the surgery.

Kim SH et al. ${ }^{[16]}$ showed that, compared with the no blood transfusion group, gastric cancer patients with postoperative blood transfusion have a poor prognosis. Some studies also showed that there is a negative correlation between the prognosis of patients and transfusion, the higher the transfusion volume, the worse the prognosis ${ }^{[17]}$. Skanberg $\mathrm{J}^{[18]}$ studied the effect of postoperative blood transfusion survival in patients with colorectal cancer and also found a relatively poor prognosis. The adverse effect of blood transfusion on prognosis has also been fully confirmed in liver cancer research ${ }^{[19]}$. Our study showed that postoperatively survival rate in patients with gastric cancer was significantly related with PABT and transfusion volume.

Although blood transfusion treatment improves anemia symptoms and the quality of life in patients with gastric cancer, it severely affects the prognosis of patients ${ }^{[20]}$. This may be related to the input of allogeneic blood which can inhibit the patients' immune function and result in a higher recurrence rate and lower survival rate ${ }^{[21]}$. Immunosuppressive effect mainly means a reduction in the activity of helper $\mathrm{T}$ cells and NK cells, as well as the monocyte/macrophage immune function declines, the activity of suppressor $\mathrm{T}$ cells increase and resistance gene antibodies are produced against antigen specific $\mathrm{T}$ cell receptors $^{[22,23]}$. As a result, blood transfusion for patients with tumors is more harm in most cases. This reminds our clinical blood transfusion workers and doctors that the blood transfusion should be taken into more careful consideration due to the specificity of tumor patients. In summary we should develop better transfusion strategies to reduce unnecessary blood transfusions.

\section{Acknowledgments}

This study was supported by the Department of Science \& Technology of Shaanxi Provincial Government (2014JM2-8195) and the First Affiliated Hospital of Xi'an Medical University (XYFY14-02). The authors express their gratitude to the study participants and research personnel for their involvement in the study. They would also like to thank Professor Ming Xie and Professor Yuqiang Ji for their valuable assistance. The authors declare that there is no conflict of competing interests regarding the publication of this article.

\section{References}

[1] Jemal A, Bray F, Center MM, et al. Global cancer statistics. CA Cancer J Clin, 2011; 61(2):69-90.

[2] Kanavos P. The rising burden of cancer in the developing world. Ann Oncol, 2006;17(S8): 15-23.

[3] Japanese Gastric Cancer Association. Japanese classification of gastric carcinoma: 3rd English edition. Gastric Cancer, 2011;14(2):101-12.

[4] Guo JR, Xu F, Jin XJ, et al. Impact of allogenic and autologous transfusion on immune function in patients with 
tumors. Asian Pac J Cancer Prev, 2014;15(1):467-74.

[5] Luan H, Ye F, Wu L, et al. Perioperative blood transfusion adversely affects prognosis after resection of lung cancer: a systematic review and a meta-analysis. $B M C$ Surg, 2014;23(14):34.

[6] Cata JP, Gottumukkala V. Blood transfusion practices in cancer surgery. Indian J Anaesth, 2014;58(5):637-42.

[7] Ojima T, Iwahashi M, Nakamori M, et al. Association of allogeneic blood transfusions and long-term survival of patients with gastric cancer after curative gastrectomy. $J$ Gastrointest Surg, 2009;13(10):1821-30.

[8] Kamei T, Kitayama J, Yamashita H, et al. Intraoperative blood loss is a critical riskfactor for peritoneal recurrence after curative resection of advancedgastric cancer. World J Surg, 2009;33(6):1240-6.

[9] Bortul M, Calligaris L, Roseano M, et al. Blood transfusions and results after curative resection for gastric cancer. Suppl Tumori, 2003;2(5): 27-30.

[10] Edge SB, Byrd DR, Carducci MA, et al. AJCC Cancer Staging Manual, 7th edition New York: SpringerVerlag, 2010.210

[11] Hallinan JT, Venkatesh SK. Gastric carcinoma: imaging diagnosis, staging and assessment of treatment response. Cancer Imaging, 2013; 30(13):212-27.

[12] Blum MA, Takashi T, Suzuki A, et al. Management of localized gastric cancer. J Surg Oncol, 2013;107(3):265-70.

[13] Rausei S, Ruspi L, Galli F, et al. Peri-operative blood transfusion in gastric cancer surgery: prognostic or confounding factor? Int J Surg, 2013;11(S1):100-3.

[14] Sahu S, Hemlata, Verma A. Adverse events related to blood transfusion. Indian J Anaesth, 2014;58(5):543-51.

[15] Isbister JP, Shander A, Spahn DR, et al. Adverse blood transfusion outcomes: establishing causation. Transfus
Med Rev, 2011;25(2):89-101.

[16] Kim SH, Lee SI, Noh SM. Prognostic significance of preoperative blood transfusion in stomach cancer. $J$ Gastric Cancer, 2010;10(4):196-205.

[17] Hyung WJ, Noh SH, Shin DW, et al. Adverse effects of perioperative transfusion on patients with stage III and IV gastric cancer, Ann Surg Oncol. 2002;9(1):5-12.

[18] Skånberg J, Lundholm K, Haglind E. Effects of blood transfusion with leucocyte depletion on length of hospital stay, respiratory assistance and survival after curative surgery for colorectal cancer. Acta Oncol, 2007;46(8):1123-30.

[19] Wang CC, Iyer SG, Low JK, et al. Perioperative factors affecting long-term outcomes of 473 consecutive patients undergoing hepatectomy for hepatocellular carcinoma, Ann Surg Oncol. 2009;16(7):1832-42.

[20] Bortul M, Calligaris L, Roseano M, et al. Blood transfusions and results after curative resection for gastric cancer, Suppl Tumori. 2003;2(5):27-30.

[21] Long MY, Liu ZH, Zhu JG. Comparative analysis of autologous blood transfusion and allogeneic blood transfusion in surgical patients. Int J Clin Exp Med, 2014;7(9):2889-94

[22] Chen G, Zhang FJ, Gong M, et al. Effect of perioperative autologous versus allogeneic blood transfusion on the immune system in gastric cancer patients. J Zhejiang Univ Sci B, 2007;8(8):560-5.

[23] Sibrowski W, Wegner W, Kühnl P. Immunomodulatory activity of different blood products on the mitogeninduced human lymphocyte transformation. Transfus Med, 1992;2(3):215-21.

(Received 8 May 2017, Revised 27 May 2017, Accepted 07 June 2017) 


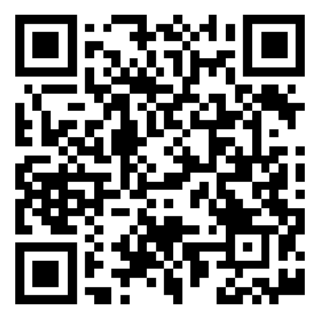

Asia-Pacific Journal

of Blood Types and Genes 